\title{
SUPERPIXEL-BASED UNSUPERVISED CHANGE DETECTION USING MULTI-DIMENSIONAL CHANGE VECTOR ANALYSIS AND SVM-BASED CLASSIFICATION
}

\author{
Zhaocong Wu, Zhongwen Hu and Qian Fan \\ School of Remote Sensing and Information Engineering \\ Wuhan University, Wuhan 430079, China \\ zcwoo@whu.edu.cn, zhongwenhu@163.com, fanqian86@gmail.com
}

Commission VII/5

KEY WORDS: Superpixel Segmentation, Simple Linear Iterative Cluster (SLIC), Unsupervised Change Detection, Multi-dimensional Change Vector, Support Vector Machines (SVM), Very High Resolution (VHR) Images

\begin{abstract}
:
In this paper, a novel superpixel-based approach is introduced for unsupervised change detection using remote sensing images. The proposed approach contains three steps: 1) Superpixel segmentation. The simple linear iterative cluster (SLIC) algorithm is applied to obtain lattice-like homogenous superpixels. To avoid discordances of the superpixel boundaries obtained from bi-temporal images, the two images are firstly fused using principle component analysis. And then, the SLIC algorithm is applied on the first three principle components, which contain the main information of the two images. 2) For each superpixel, which is considered as the basic unit of the image space, the multi-dimensional change vector is computed from spectral, textural and structural features. 3) The superpixels are classified into two type: changed and unchanged through two progressive classification processes. The superpixels are firstly cataloged into three types: changed, unchanged and undefined by thresholding the change vectors and a voting process. And then the undefined superpixels are further classified into two classes: changed and unchanged, using a SVM-based classifier, which is trained by the derived changed and unchanged superpixels from the former step. The experiment using Indonesia data set has confirmed that the proposed approach is able to detect the changes automatically, by exploiting multiple change features.
\end{abstract}

\section{INTRODUCTION}

In remote sensing applications, change detection is the process of identifying differences in the state of an object or phenomenon by analyzing a pair of images acquired on the same geographical area at two different instants (Singh, 1989). It is one of the most important applications of the automatic analysis of multitemporal remote sensing images, and has been widely used in many fields, such as forest managements, urban planning, and disaster evaluation. In the past few years, many methods have been developed, which can be simply cataloged into two types: pixel-based and object-oriented, differing in the basic unit utilized.

Numerous pixel-based change detection algorithms have been developed using low and medium resolution remote sensing images. Some of these include post-classification comparison (J.Im and Jensen, 2005), image algebra (Bruzzone and Prieto, 2000), multiple-date composite image change detection using principal component analysis (Deng et al., 2008, Celik, 2009), and spectral change vector analysis (Johnson and Kasischke, 1998). In addition, many optima algorithms and techniques have also been used to improve the qualities and efficiencies, such as neural networks (Ghosh et al., 2007), genetic algorithm (Celik, 2010) and support vector machines (Bovolo et al., 2008). The pixel-based approaches are theoretically simple and perform well for low and medium resolution imagery. However, the pixels in the high-resolution images, are not spatially independent, which makes the conventional pixel-based change detection techniques less effective.

Compared with pixel-based method, the object-oriented approach is a recently developed knowledge-based technique. It considers landscapes as aggregations of meaningful objects corresponding to ground entities and patches of surface cover (Dronova et al.,
2011). In general, it starts with a segmentation process, and is followed by a successive analysis with the help of expert knowledge. It represents a obvious advantage in analyzing high-resolution data because image pixels are meaningfully grouped into networked homogeneous objects, and the noises are consequently reduced (Lu et al., 2011). In addition, it is efficient in combining multiple features, such as spectral (Hazel, 2001, Huo et al., 2010, Tang et al., 2011), textural (Walter, 2004) and contextual (J.Im et al., 2008) features. However, there're still many obstacles for unsupervised and automatic object-oriented change detection, because this kind of methods largely depend on the image segmentation, and expert knowledge.

In this paper, a novel superpixel-based approach is proposed for unsupervised and automatic change detection. A super-pixel is a set of pixels which have similar spectrum and are adjacent in space. Its usually highly homogeneous, uniform and compact, and an object is usually composed of several super-pixels. The approach consists of three steps: 1) Superpixel segmentation. The bi-temporal images are firstly partitioned into evenly distributed compact and homogeneous superpixels. To avoid discordances of superpixel boundaries obtained from bi-temporal images, the images are fused using principle components analysis, and only one superpixle map is obtained. 2) Multi-dimensional change vector extraction. Superpixel level spectral, textural and spatial differences are calculated to measure the changes comprehensively. 3) Superpixel level change classification. In this step, two progressive classification processes are applied. The superpixels are firstly classified into three types (changed, unchanged and undefined) by thresholding the change vectors. And then, the undefined superpixels are going through a further classification based on a SVM-based classifier, which is trained by the derived changed and unchanged superpixels from the former step.

The proposed approach is expected to take advantages of both 
the pixel-based and the object-based approaches. Firstly, the even distribution of homogenous superpixels retains the statistical characteristics of changed and unchanged pixels. It enable us to use the statistical tools for automatical analysis, which have been widely used in pixel-based approaches. Secondly, it considers the superpixel as the smallest unit, which enables us to model the changes in an object (region) -based manner, where region-based spectral, textural and structural differences can be utilized.

\section{METHODOLOGY}

In the proposed method, the reference and changed images are pre-registered and radiometric corrected. The work flow of the method can be simple divided into three steps: superpixel segmentation, multi-dimensional change vector extraction and unsupervised progressive classification, where the last step can be divided into two sub-steps. The work flow of the method is presented in Figure.1 .
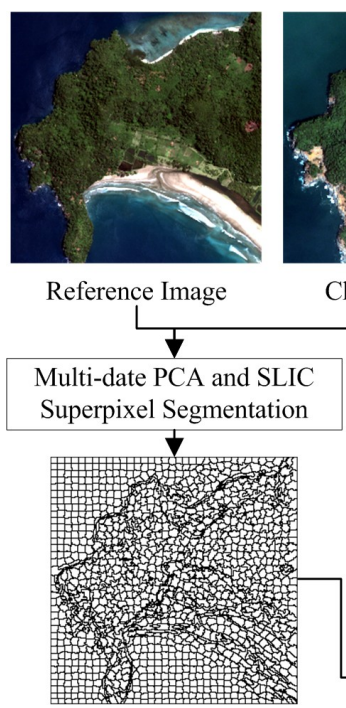

Superpixel map

Figure 1: The flowchart of the proposed method.

\subsection{SLIC Superpixel Segmentation}

In this step, the simple iterative cluster (SLIC) (Achanta et al., 2010) algorithm is utilized to obtain superpixel map. To avoid any discordances of the superpixel boundaries obtained from the bi-temporal images, only one superpixel map is needed. In our method, the layers of bi-temporal images are stacked together. And then, the principle components analysis is applied to remove the redundant information and only the first three principle components are selected for SLIC algorithm.

SLIC segmentation is an efficient cluster technique, which is similar to K-means cluster method. It starts with sampling $\mathrm{K}$ regularly spaced cluster centers, followed by a k-means clustering process. More specially, the clustering distance between two different pixels is weighted by the color distance and space distance. The color distance ensures the superpixel homogeneous, and the spatial constraint force the superpixel compact. The color distances of two pixels $m$ and $n$ is defined as follows:

$$
d_{\text {color }}=\sqrt{\sum_{i=0}^{N-1}\left(X_{m}^{i}-X_{n}^{i}\right)^{2}}
$$

where $\mathrm{N}$ denotes the number of spectral channels; $X^{i}$ denotes the gray value in the $i-$ th channel. The space distance is defined as:

$$
d_{x y}=\sqrt{\left(x_{m}-x_{n}\right)^{2}+\left(y_{m}-y_{n}\right)^{2}}
$$

where $x$ and $y$ denote the location of the pixel. And then, the weighted distance is defined as:

$$
d_{m, n}=d_{\text {color }}+\frac{m}{s} d_{x y}
$$

where $s$ is the width of grids; and $m$ is a variable introduced in $d_{m, n}$ to control the compactness of superpixels. The greater the value of $m$, the more spatial proximity is emphasized and the more compact the cluster. The parameter $s$ controls the size of the superpixels, the greater value of $s$ : the larger the superpixels.

The details of the SLIC algorithm is described in Algorithm.1:

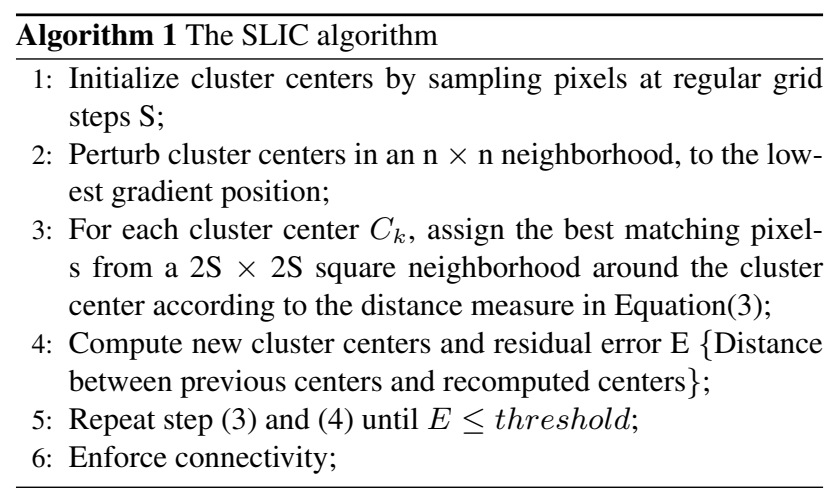

\subsection{Superpixel-based Change Vector Extraction}

The change detection is achieved by analyzing the change vectors of the superpixels. One of the most popular methods is change vector analysis (CVA) (Johnson and Kasischke, 1998). It is effective in combining different kinds of features to detect changes. This technique has been widely used in many change detection methods, such as the works listed in the references (Johnson and Kasischke, 1998, Bovolo, 2009, Huo et al., 2010, He et al., 2011). However, the dominant limitation of these methods are their too heavy reliance on the spectral features. They only consider the spectral values of pixels to construct the change vectors. Since the pixels are not spatial independent, differences on spectral means may fail to reveal the changes in high resolution images. In our method, a 5-dimensional change vector containing spectral, textural and structural features are employed to model the changes between two images.

- Spectral difference: In most cases, changes are mainly characterized by the gray value changes. In this method, the spectral differences of each superpixels are characterized by the Euclidean distance and the fused distance of two superpixel. The Euclidean distance measures the changes of spectral means between multi-temporal images, it is defined as:

$$
d_{\text {Euclidean }}=\sqrt{\sum_{i=1}^{N}\left(\mu_{1}^{i}-\mu_{2}^{i}\right)^{2}}
$$

where $\mathrm{N}$ denotes the number of spectral channels; $\mu_{1}^{i}$ and $\mu_{2}^{i}$ represent the mean values of $i$-th spectral channel at two different dates respectively. The fused distance considers the data distribution of pixel values in the superpixel, and measures the difference with the changes of standard deviations. It is defined 
as follows:

$$
d_{\text {Fused }}=\sqrt{\sum_{i=1}^{N}\left(2 \sigma_{\text {Fused }}^{i}-\left(\sigma_{1}^{i}+\sigma_{2}^{i}\right)\right)^{2}}
$$

where $\sigma_{1}^{i}$ and $\sigma_{2}^{i}$ denote the $i$-th standard deviations of this superpixel on the reference image and changed image; $\sigma_{F u s e d}^{i}$ is the $i$-th standard deviation when the pixel values at two different dates are viewed as one data set.

- Textural difference: Different land-cover classes cannot be distinguished only by their spectral behavior but also by their $\mathrm{d}$ ifferent textures. In this proposed method, we measure the texture differences of bi-temporal images using the distance of joint histogram of local binary patterns (LBP) (Ojala et al., 1996) and contrast. For each superpixel, the LBP and contrast at two different dates are calculated separately, and then their LBP and contrast histograms are computed. Finally, the nonparametric G-statistic technique (Ojala et al., 1996) is utilized to measure the distance.

$$
\left\{\begin{array}{l}
d_{\text {textural }}= \\
\sum_{t_{1}, t_{2}} \sum_{i=1}^{n} f_{i} \log f_{i}+\left(\sum_{t_{1}, t_{2}} \sum_{i=1}^{n} f_{i}\right) \log \left(\sum_{t_{1}, t_{2}} \sum_{i=1}^{n} f_{i}\right) \\
-\sum_{t_{1}, t_{2}}\left(\sum_{i=1}^{n} f_{i}\right) \log \left(\sum_{i=0}^{t-1} f_{i}\right)-\sum_{i=1}^{n}\left(\sum_{t_{1}, t_{2}} f_{i}\right) \log \left(\sum_{t_{1}, t_{2}} f_{i}\right)
\end{array}\right\}
$$

where $t_{1}$ and $t_{2}$ denote two different dates; $f_{i}$ denotes the corresponding value at the $i$-th bin in the histograms; n denotes number of bins; $d_{\text {textural }}$ denotes the corresponding textural difference between two dates.

- Structural difference: The structural differences of superpixel$s$ are characterized at two scales: the pixel level correlation and the superpixel level correlation. The pixel-level correlation measures the structural consistency of pixels in a superpixel at two different dates. It is defined as follows:

$$
r_{t_{1}, t_{2}}=\frac{\sum_{i=1}^{m}\left(x_{t_{1}}^{i}-\mu_{t_{1}}\right)\left(x_{t_{2}}^{i}-\mu_{t_{2}}\right)}{\sqrt{\sum_{i=1}^{m}\left(x_{t_{1}}^{i}-\mu_{t_{1}}\right)^{2}} \sqrt{\sum_{i=1}^{m}\left(x_{t_{2}}^{i}-\mu_{t_{2}}\right)^{2}}}
$$

where $t_{1}$ and $t_{2}$ denote the two different dates; $m$ denotes the number of pixels in the superpixel; $x_{t_{1}}$ and $x_{t_{2}}$ denote the pixel values at $t_{1}$ and $t_{2}$ respectively; and $\mu$ denotes the mean value.

The superpixel level correlation describes the structural consistency at a coarser scale. It considers each superpixel as a single point, and then the correlation is calculated based on the superpixel's and its neighborhoods' mean values by Equation (7). It is worth mentioning that the symbol $m$ used in Equation (7) denotes the number of superpixels used here, including the superpixel and its neighborhoods.

\subsection{Progressive Superpixel Classification}

In this step, we are aiming at classifying the superpixels into two types: changed and unchanged, by analogizing the derived change vector. The CVA technique is a powerful tool for this task. However, since it is a supervised technique, reliable changed and unchanged samples are needed. In this section, two progressive steps are utilized to achieve a fully automatic classification of superpixels.

2.3.1 Change Vector Thresholding and Voting The thresholding of change vector is the simplest tool to classify the su- perpixels. This kind of methods have been widely used in pixelbased change detection applications. Several unsupervised thresholding methods have been proposed for this task, suche as EM algorithm (Bruzzone and Prieto, 2000), Genetic Algorithm (GA) (Celik, 2010), K\&I technique (Kittler and Illingworth, 1986). Among which, the K\&I threshold method is one of the most widely used method (Moser and Serpico, 2006, Patra et al., 2011). It considers the thresholding of the image as a two-category classification problem, and aims at minimizing the probability of classification error (Kittler and Illingworth, 1986)

In this method, the histogram is viewed as an estimation of the probability density function of the mixture population for the changed and unchanged pixels. It is assumed that the changed and unchanged pixels are normally distributed with $N\left(\mu_{c}, \sigma_{c}\right)$ and $N\left(\mu_{u}, \sigma_{u}\right)$. Then, the total error of the thresholding by $t$ is given as:

$$
E(t)=\int_{-\infty}^{t} f_{c}(x) d x+\int_{t}^{+\infty} f_{u}(x) d x
$$

where $f_{c}(x)$ and $f_{u}(x)$ denote the probability density functions of the changed and unchanged superpixels.

To find out the minimum error threshold $t$, instead of solving the above quadratic equation, Kittler and Illingworth defined a criterion function $J(t)$ for threshold $t$ as follows:

$$
J(t)=1+2\left(P_{u} \log \sigma_{u}+P_{c} \log \sigma_{c}\right)-2\left(P_{u} \log P_{u}+P_{c} \log P_{c}\right)
$$

where

$$
\begin{aligned}
\sigma_{c} & =\sqrt{\frac{1}{P_{c}} \sum_{i=t+1}^{L-1}\left(i-\mu_{c}\right)^{2} f(i)} \\
\sigma_{u} & =\sqrt{\frac{1}{P_{u}} \sum_{i=0}^{t}\left(i-\mu_{u}\right)^{2} f(i)}
\end{aligned}
$$

where $P_{c}$ and $P_{u}$ are the prior probabilities of changed and unchanged superpixels, respectively. Then the optimal threshold $t$ is obtained by minimizing $J(t)$,

$$
t=\min \{J(t)\}
$$

By applying the K\&I thresholding on the 5-dimensional change vector, a 5-dimensional Change Indicator (CI) is obtained. And for each superpixel, there are 5 tickets indicating whether it prior changed. Then, a voting process is employed, in which the superpixels with 0 ticket is classified as unchanged, and superpixels with no less than 3 tickets is cataloged as changed, otherwise undefined. The class of the $i$-th superpixel is defined as follows:

$$
C_{i}=\left\{\begin{aligned}
0 & \text { (unchanged sample }), \quad T_{i}=0 \\
-1 & (\text { undefined }), \quad 1 \leq T_{i} \leq 2 \\
1 & \text { (changed sample }), \quad T_{i} \geq 3
\end{aligned}\right.
$$

where $T_{i}=\sum_{j=1}^{5} C I_{i}^{j}$ denotes the total number of the tickets for this superpixel.

2.3.2 SVM-based Classification Here, reliable changed and unchanged samples have been obtained. However, there still exist the uncertain superpiexls which are not classified and need further analysis, thus a SVM-based classification process is employed. SVM is a statistic classification method proposed by Cortes (Cortes and Vapnik, 1995). It addresses the small sample, nonlinear and high dimensional pattern recognition problems and is originally designed for binary classification. It is a distributionfree classifier and fits the requirement of change detection clas- 
sification problem, especially when the changed areas is very small compared to the whole image. The SVM-based classifier is trained using the derived changed and unchanged superpixels based on their change vectors. And finally, the uncertain superpixels are classified into changed and unchanged ones depending on their change vectors.

\section{CASE STUDY AND DISCUSSIONS}

To evaluate the proposed approach, experiments were conducted on several different sets of satellite images, including several VHR images and medium spatial resolution images. Because of the limitation of paper length, only the results of one case study area using QuickBird images are shown in this paper.

\subsection{Data set description}

The study area is located in Indonesia that was impacted by tsunami on the December 26, 2004. The reference image was acquired in April, 2004 and the other image was taken in January, 2005. Each of these two images contains four raster channels (R,G,B and NIR), having $3217 * 4231$ pixels with a resolution of $2.4 \mathrm{~m}$. It can be easily observed from Figure. 2 that substantial differences exist between the two images. Many vegetation and agricultural areas were washed out by the tsunami. More specifically, some areas are covered by clouds.

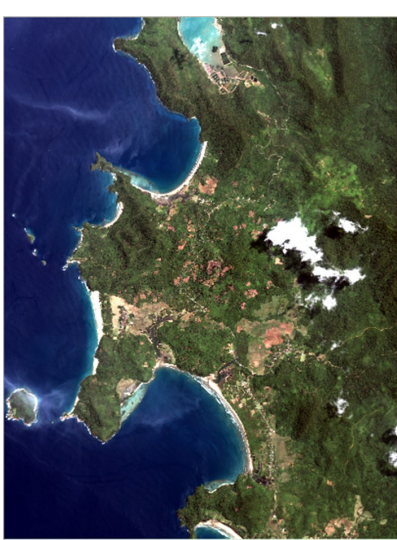

(a)

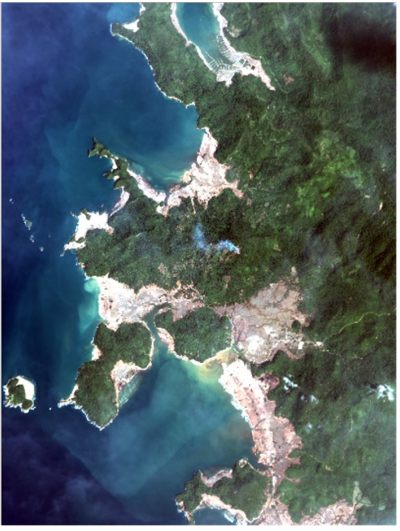

(b)
Figure 2: The test Quickbird images $(3217 \times 4231$ pixels, $2.4 \mathrm{~m}$ resolution): (a) Image acquired before tsunami; (b) Image acquired after tsunami.

\subsection{Superpixel segmentation}

The test images were fused using PCA and segmented using the SLIC algorithm. The parameters of SLIC algorithm were set as $m=30, s=15$. A subset of the superpixel map is presented in Figure.3.

As are shown in the figures, the lattice-like superpixels have been obtained, which are highly homogenous, compact and evenly distributed. More specially, since the SLIC algorithm was applied on the first three PCs, which contain the main information of the bi-temporal images, the boundaries of the superpixels are coincided with the edges of both the two images. On the other hand, the evenly distributed superpixels will not dramatically disturb the distribution of changed and unchange pixels, which is very important for many unsupervised thresholding techniques.

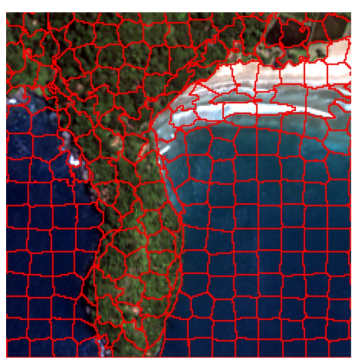

(a)

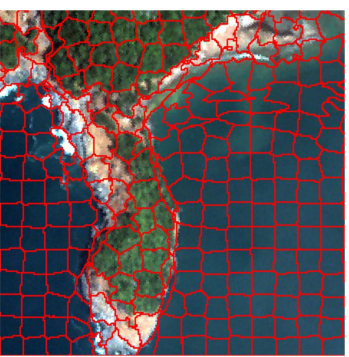

(b)
Figure 3: Superpixel map (a subset of the image), $m=30, s=$ 15. (a) Superpixel map overlap on the reference image. (b) Superpixel map overlap on the changed image.

\subsection{Change detection results}

The superpixel-based spectral, texture and spatial differences are calculated. And then, each dimension of the change vector is thresholded, and the superpixels are classified into three types through a voting process: changed, unchanged and undefined. Finally, the SVM-based classification procedure is conducted to analyze the uncertain superpixels and obtain the changed map. The change detection result of the whole area is presented in Figure.4.

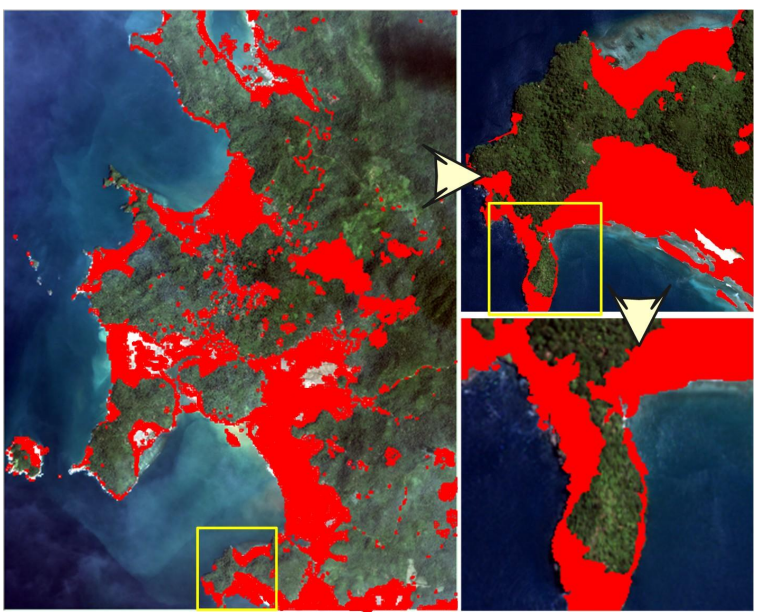

Figure 4: Change detection results of the whole image. (left: overview of the result; right: the zoomed view of the result.

In order to analyze the proposed method in a quantitative way, a subset of the images are used to test different methods. Experiments using pure spectral, textural and spatial features are carried out. In addition, the proposed approach is compared with the traditional pixel-based approach and the object-oriented change detection approach. The pixel-based approach is carried out by applying the K\&I technique on the magnitude of the spectral $\mathrm{d}$ ifferences between images. It is a pure spectral-based change detection technique. For the object-oriented approach, one segmentation results is obtained using the bi-temporal image segmentation presented in (Huo et al., 2010), and then the change vectors are extracted using object level spectral, textural and spatial features. More importantly, some changed and unchanged samples are selected manually to train the SVM classifier.

The subset of images and reference change map are presented in Figure. 5. The change detection results are presented in Figure.6. The quantitative analysis of these methods by comparing with the reference map are shown in Table.1. 


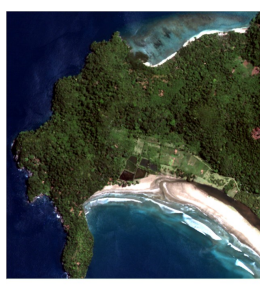

(a)

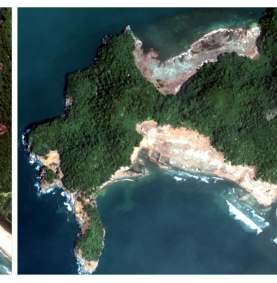

(b)

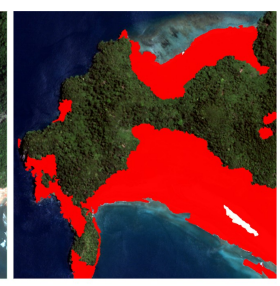

(c)
Figure 5: A subset of the image $(532 \times 550$ pixels): (a) Image acquired before tsunami; (b) Image acquired after tsunami; (c) Reference changes.

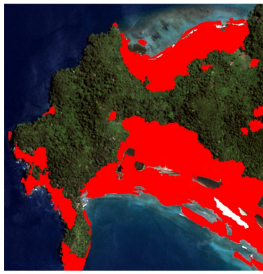

(a)

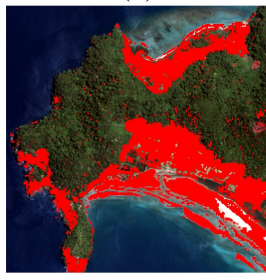

(d)

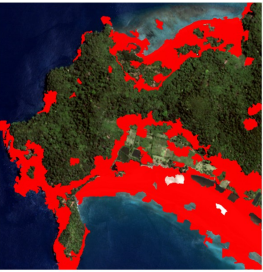

(b)

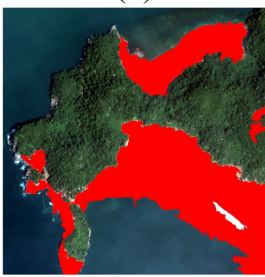

(e)

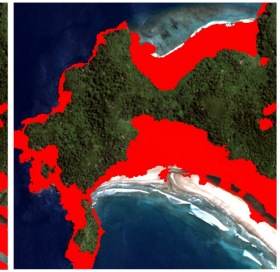

(c)

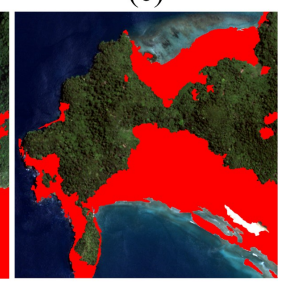

(f)
Figure 6: Change detection results: (a) Change detection using only spectral features; (b) Change detection using only textural features; (c) Change detection using only spatial features; (d)Pixel-based approach; (e) Object-oriented approach; (f) The proposed approach

The Figure.6 (a) illustrates the change detection result obtained from using only spectral differences. As is shown in the figure, most changes have been correctly detected, with a high kappa accuracy of 0.95 . However, several changed areas are still missed. The Figure.6 (b) illustrates the change detection result using textural difference. It can be seen that the textural indicator can well measure the textural differences of two images, especially for the superpixels around the boundaries of the changed and unchanged areas. But it is unable to measure the differences of the areas with similar texture but different spectral features, which could result in many missed alarms and the lowest kappa accuracy of five methods. The change detection results using only spatial correlations is presented in Figure.6 (c). It can be seen that the washed farmland and forest areas are properly detected, but the changed sea beach areas are missed. That's mainly because the spatial pattern of these areas at two different times are highly correlated, despite of their different spectral and textural features.

As is shown in Figure.6 (d), the pixel-based change detection approach is able to detect most of the changes with a low false alar$\mathrm{m}$ rate. However, there are still many changed areas undetected. In addition, many isolated pixels are mistakenly cataloged as changed ones, which are usually considered as noises. The object-oriented change detection result is presented in Figure.6 (e). As is shown in the figure, the object-oriented change detection holds the best kappa accuracy (0.9617), that's because the changed and unchanged samples are chosen manually. Although the object-oriented approach hold the best kappa accuracy, this

\begin{tabular}{ccccc}
\hline & $\begin{array}{c}\text { False } \\
\text { alarms }\end{array}$ & $\begin{array}{c}\text { Missed } \\
\text { alarms }\end{array}$ & $\begin{array}{c}\text { Overall } \\
\text { errors }\end{array}$ & $\begin{array}{c}\text { Kappa } \\
\text { accuracy }\end{array}$ \\
\hline Spectral & 1972 & 12455 & 14427 & 0.9501 \\
Textural & 13601 & 33060 & 46664 & 0.8364 \\
Structural & 7874 & 26850 & 34724 & 0.8783 \\
Pixel-based & 4041 & 23780 & 27821 & 0.9024 \\
Object-based & 3531 & 7643 & 11174 & 0.9617 \\
Proposed & 5717 & 6667 & 12381 & 0.9576 \\
\hline
\end{tabular}

Table 1: False alarms, missed alarms, overall errors and kappa accuracy of the change detection results of the test site.

approach needs lots of human interventions in selecting samples. We had to choose samples of different changing types and this is time consuming.

Change detection result using the proposed method is presented in Figure.6 (e). As is shown in the figure, change detection using multiple-features combined indicator performs much better than the results using single change indicator. That's because the method can make full use of the spectral, textural and spatial differences comprehensively to detect the changes. As are shown in the figures, the superpixel-based approach performs better than the pixel-wise approach, since it has overcome the salt and pepper noises and get changed map with no isolated pixels. The result obtained by superpixel-based approach is similar to the result obtained using object-oriented approach, the kappa accuracy ranks the second in all of the six method. However, the proposed method is a totally unsupervised change detection method, it's effective and efficient.

\section{CONCLUSIONS AND FUTURE WORK}

In this work, a novel superpixel-based unsupervised change detection approach is proposed. The advantages of the proposed approach lie in the following three aspects:

- The superpixels enable us to overcome the limitation of pixelbased approaches and calculate object (region) -based change indicators, by exploiting spectral, textural and spatial features. On the other hand, the uniformity and evenly distribution of the superpixels make the statistic of the changed and unchanged superpixels stable and reliable. And many statistical methods can be utilized for automatic analysis of the changes, which have been widely used in pixel-wise approaches.

- The approach takes a superpixel as the basic unit, which makes the approach not sensitive to the register errors, as well as to the salt and pepper noises.

- The superpixel-based approach can reduce the number of change vectors dramatically, which will bring a great efficiency improvement. The proposed method could complete the calculation in less than five minutes on the Indonesia data set with a personal computer equipped with 2-GB RAM and Intel Core 2 Duo 2.83-GHz processor. But for the normal pixel-based change vector analysis and SVM-based classification procedures, it will take several hours for this task.

Despite of the promising preliminary results, many works are still needed. In this paper, we adopted the K\&I method for thresholding of the changed vector. It defines a criterion based on the assumption that the histograms of the changed and unchanged pixels are normally distributed. And then a threshold is selected by minimizing this criterion, the smaller the overlap between the density functions of the changed and unchanged superpixels, 
the lower is the total error. However, when the changed and unchanged pixels are not normally distributed, the K\&I thresholding method may failed to find the correct threshold. In addition, when the changed pixels take only a very small part of the whole image, they will probably be clustered only in several superpixels, and this will lead to the failure of the thresholding technique. Thus, studies on the distribution-free thresholding technique are needed in the future.

The superpixel is a set of homogenous pixels, and can be regarded as the linkage of the single pixels to the meaningful objects. It provides us the outline of pixels-superpixels-objects to analysis the multi-temporal images. Our future work will focus on the multi-scale analysis of multi-temporal images using the pixelssuperpixels-objects architecture.

\section{REFERENCES}

Achanta, R., Shaji, A., Smith, K., Lucchi, A. and Susstrunk, S., 2010. Slic superpixels. EPFL Technical Report 149300.

Bovolo, F., 2009. A multilevel parel-based approach to change detection in very high resolution multitemporal images. IEEE Geoscience and Remote Sensing Letters 6(1), pp. 33-37.

Bovolo, F., Bruzzone, L. and Marconcini, M., 2008. A novel approach to unsupervised change detection based on a semisupervised svm and a similarity measure. IEEE Geoscience and Remote Sensing 46(7), pp. 2070-2082.

Bruzzone, L. and Prieto, D. F., 2000. Automatic analysis of difference image for unsupervised change detection. IEEE Transactions on Geoscience and Remote Sensing 38(3), pp. 1171-1182.

Celik, T., 2009. Unsupervised change detection in satellite images using principal component analysis and k-means clustering. IEEE Geoscience and Remote Sensing Letters 6(4), pp. 772-776.

Celik, T., 2010. Change detection in satellite images using a genetic algorithm approach. IEEE Geoscience and Remote Sensing Letters 7(2), pp. 386-390.

Cortes, C. and Vapnik, V., 1995. Support vector networks. Machine Learning 20, pp. 273-297.

Deng, J. S., Wang, K., Deng, Y. H. and Qi, G. J., 2008. Pca-based land-use change detection and analysis using multitemporal and multisensor satellite data. International Journal of Remote Sensing 29(16), pp. 4823-4838.

Dronova, I., Gong, P. and Wang, L., 2011. Object-based analysis and change detection of major wetland cover types and their classification uncertainty during the low water period at poyang lake, china. Remote Sensing of Enviroment(Article in press).

Ghosh, S., Bruzzone, L., Patra, S., Bovolo, F. and Ghosh, A., 2007. A context-sensitive technique for unsupervised change detection based on hopfield-type neural networks. IEEE Transactions on Geoscience and Remote Sensing 45(3), pp. 778-789.
Hazel, G. G., 2001. Object-level change detection in spectral imagery. IEEE Transactions on Geoscience and Remote Sensing 39(3), pp. 553-561.

He, C., Wei, A., Shi, P., Zhang, Q. and Zhao, Y., 2011. Detecting land-use/land-cover change in rural-urban fringe areas using extended change vecor analysis. International Journal of Applied Earth Observation and Geoinformation 13, pp. 572-585.

Huo, C., Zhou, Z., Lu, H., Pan, C. and Chen, K., 2010. Fast object-level change detection for vhr images. IEEE Geoscience and Remote Sensing Letters 7(1), pp. 118-122.

J.Im and Jensen, J., 2005. A change detection model based on neighborhood correlation image analysis and decision tree classification. Remote Sensing of Enviroment 99, pp. 326-340.

J.Im, Jensen, J. and Tullis, J., 2008. Object-based change detection using correlation image analysis and image segmentation. International Journal of Remote Sensing 29(2), pp. 399-423.

Johnson, R. D. and Kasischke, E. S., 1998. Change vector analysis: A technique for the multispectral monitoring of land cover and condition. International Journal of Remote Sensing 19(3), pp. 411-426.

Kittler, J. and Illingworth, J., 1986. Minimum error thresholding. Patter Recognition 19, pp. 41-47.

Lu, P., Stumpf, A., Kerle, N. and Casagli, N., 2011. Objectoriented change detection for landslide rapid mapping. IEEE Geoscience and Remote Sensing Letters 8(4), pp. 701-705.

Moser, G. and Serpico, S. B., 2006. Generalized minimum-error thresholding for unsupervised change detection from sar amplitude imagery. IEEE Transactions on Geoscience and Remote Sensing 44(10), pp. 2972-2982.

Ojala, T., Pietikainen, M. and Harwood, D., 1996. A comparative study of texture measures with classification based on feature distributions. Pattern Recognition 29(1), pp. 51-59.

Patra, S., Ghosh, S. and Ghosh, A., 2011. Histogram thresholding for unsupervised change detection of remote sensing images. International Journal of Remote Sensing 32(21), pp. 6071-6089.

Singh, A., 1989. Digital change detection techniques using remotely sensed data. International Journal of Remote Sensing pp. 989-1003.

Tang, Y., Zhang, L. and Huang, X., 2011. Object-oriented change detection based on kolmogorov-smirnov test using highresolution multispetral imagery. International Journal of Remote Sensing 32(20), pp. 5719-5740.

Walter, V., 2004. Object-based classification of remote sensing data for change detection. ISPRS Journal of Photogrammety \& Remote Sensing 58, pp. 225-238. 